\title{
Controlling Tumor Regression about the Physiological System
}

\begin{abstract}
Makrushin AV*
ID Papanin Institute for Biology of Inland Waters Russian Academy of Science, Russia

*Correspondling author: AV Makrushin, ID Papanin Institute for Biology of Inland Waters Russian Academy of Science, 152742. Yaroslavskaya oblast, Borok, Russia
\end{abstract}

Received: August 05, 2021; Accepted: August 19, 2021; Published: August 26, 2021

\begin{abstract}
It is proposed to study the mechanism of tumor regression from an evolutionary point of view. The proposal is based on the assumption that the evolutionary precursor of this mechanism is the resorption mechanism in sedentary modular invertebrates preparing for diapause in embryos. To understand how the tumor regression mechanism works, it is necessary to investigate the mechanism of resorption of these embryos in these invertebrates.
\end{abstract}

Keywords: Cancer pathogenesis, Apical dominance, Modular invertebrates, Evolution of Metazoa.

\section{Introduction}

The pathogenesis of cancer is not yet clear. Most oncologists believe that a malignant tumor arises from the accumulation of mutations in one single cell, which, multiplying, creates a tumor. Guided by this understanding of the nature of cancer, oncologists still have not been able to offer a reliable cure for this disease. The mechanism of tumor initiation is probably different from what they think. Which? Oncogenesis is similar to the transition to diapause of sessile modular invertebrates-sponges, hydroid and coral polyps, bryozoans, intra-powder and colonial ascidians. These invertebrates diapause during the early embryo stage. But embryos formed not from a zygote, but from a set of somatic cells. Such embryos are formed by asexual reproduction. They are called somatic embryos, or kidneys. Asexual reproduction is the formation of new individuals from a set of somatic cells. Information about asexual reproduction of animals and the processes of formation of somatic diapause embryos in sedentary modular invertebrates is collected in the book by O.I. Ivanova-Kazas [1].

Here are some of the similarities of oncogenesis with the transition to diapause of these invertebrates. 1) A patient learns that he has cancer when he is diagnosed with a malignant tumor. A tumor is a structure consisting of malignant, that is, dedifferentiated cells. The transition of these invertebrates to diapause also begins with the appearance in the body of a modular organism of structures consisting of dedifferentiated cells. These structures are the rudiments of embryos preparing for diapause. 2) Tumor growth is the multiplication of dedifferentiated cells. Preparation for diapause of these invertebrates is also the reproduction of dedifferentiated cells that make up the rudiments of diapause embryos. 3) The growing tumor takes nutrients from the healthy tissues of the patient. The growing embryos preparing for diapause also take nutrients from the tissues of the modular invertebrate that is turning into diapause. Therefore, tumor growth is accompanied by depletion of an organism with cancer, and the growth of embryos preparing for diapause is accompanied by depletion of the modular invertebrate organism passing into embryonic diapause. 4) As a result of exhaustion, the body of the cancer patient and the body of the modular invertebrate are destroyed. The destruction of the body of the patient with cancer leads to his death. The destruction of the organism of the modular invertebrate, which has given all the nutrients in it to the diapausing embryos, also leads to its death. These similarities need explanation. and the growth of embryos preparing for diapause is accompanied by depletion of the modular invertebrate organism, which passes into embryonic diapause. 4) As a result of exhaustion, the body of the cancer patient and the body of the modular invertebrate are destroyed. The destruction of the body of the patient with cancer leads to his death. The destruction of the organism of a modular invertebrate, which has given all the nutrients in it to diapausing embryos, also leads to its death. These similarities need explanation. and the growth of embryos preparing for diapause is accompanied by depletion of the modular invertebrate organism, which passes into embryonic diapause. 4) As a result of exhaustion, the body of the cancer patient and the body of the modular invertebrate are destroyed. The destruction of the body of the patient with cancer leads to his death. The destruction of the organism of the modular invertebrate, which has given all the nutrients in it to the diapausing embryos, also leads to its death. These similarities need explanation. giving all the nutrients in it to diapausing embryos also leads to his death. These similarities need explanation. giving all the nutrients in it to diapausing embryos also leads to his death. These similarities need explanation.

There is an opinion that the first Metazoa on Earth were sedentary and modular [2-6]. If this is indeed the case, then the ancestors of all currently existing Metazoa diapause at the stage of early somatic embryos and their transition to diapause proceeded in the same way as in modern sessile modular invertebrates. Oncogenesis is probably the result of the inclusion of the ancient mechanism of preparation for embryonic diapause, inherited from the Precambrian ancestors of all Metazoa, and the tumor is an atavistic somatic embryo preparing for diapause. The processes of destruction of the organism accompanying oncogenesis are atavistic adaptive processes of destruction of the organism that accompanied the transition to embryonic diapause in the first Metazoa on Earth [7]. If this explanation of the similarity of oncogenesis with the transition to diapause of the aforementioned invertebrates is correct, then cancer in sedentary modular invertebrates is impossible. In them, the mechanism of formation of diapausing embryos has not yet turned 
into oncogenesis. It follows from the foregoing that tumor regression is an atavistic process of resorption of a growing somatic embryo preparing for diapause. Resorption of growing embryos preparing for diapause in sessile modular invertebrates and tumor regression are probably the same process. It only manifests itself in different ways in the most low-integrated and in the rest of Metazoa. Since resorption in sedentary modular invertebrates of embryos preparing for diapause is an evolutionary precursor of tumor regression, in order to understand how the regulatory physiological system works, controlling tumor regression, it is necessary to understand how the physiological regulatory system worked, which controlled the resorption of embryos preparing for diapause in the Precambrian sessile modular ancestors of all Metazoa. The purpose of this article is to discuss how this regulatory system works in modern sessile modular invertebrates.

Resorption of growing embryos preparing for diapause has been described in modular ascidians [8,9]. Probably, in the other invertebrates mentioned above, preparation for diapause is sometimes interrupted and embryos preparing to experience an unfavorable season for life are absorbed. ANDI do not know of the studies in sedentary modular invertebrates of the regulatory physiological system that controls the resorption of embryos preparing for diapause. I am also unaware of studies of the physiological system that controls the formation of embryos preparing for diapause in these invertebrates. Probably they were not carried out. Therefore, the discussion will have to use inferences based on analogies.

\section{The Role of Interrupting Diapause Preparation}

Why is the beginning preparation for the seasonal deterioration of the environment in sedentary modular invertebrates sometimes interrupted? Why do these invertebrates of embryos preparing for diapause, for the formation of which the body's resources were spent, sometimes dissolve? Let's discuss these issues from an environmental point of view. The transition of the colony of these invertebrates from the formation of embryos developing without diapause (from which actively functioning zooids of a modular organism are formed) to the formation of diapausing embryos occurs in response to signaling factors warning of an impending deterioration of the environment. These factors can be various changes in nature, not necessarily having a negative effect on the metabolism of an individual, but always preceding a change in the environment that has a strong negative effect on its metabolism. A signal factor that warns of the imminent freezing of a reservoir is a reduction in the duration of daylight. A signal factor that warns of the imminent drying of a rain puddle is the accumulation of hydrobionts in it and an increase in the concentration of dissolved substances in the water.

They warn of the inevitable deterioration of the environment in aquatic organisms living in those reservoirs in which the water temperature in autumn drops to values that do not allow them to exist in an active state inevitably. In winter, these reservoirs are covered with ice and are freed from it only in spring. The situation is different in water bodies located at a geographical latitude, where the climate is mild. There, a decrease in the duration of daylight, causing the colony to enter diapause, warns of a possible imminent strong drop in water temperature, which may or may not occur. At these latitudes in reservoirs, the water temperature in winter does not always drop to values that do not allow their inhabitants to exist in an active state. Some winters are warm there. Probably, in these reservoirs, after the action of the signaling factor, that warned aquatic organisms about the approach of winter and forced them to prepare for diapause, some other signaling factor acts, warning that winter will not be severe and therefore there is no need to switch to diapause. In response to this second putative signaling factor, the hydrobionts of the embryos preparing for diapause dissolve. In the rain puddles drying up in summer, in which aquatic organisms begin to prepare for diapause, the situation is also different than in water bodies in which the deterioration of the environment in the fall is inevitable. Living conditions in drying puddles can improve if rain falls and the puddle becomes full again. Then the invertebrates of the embryos preparing for diapause, which have begun preparation for diapause, dissolve. That winter will not be harsh and therefore there is no need to switch to diapause. In response to this second putative signaling factor, the hydrobionts of the embryos preparing for diapause dissolve. In the rain puddles drying up in summer, in which aquatic organisms begin to prepare for diapause, the situation is also different than in water bodies in which the deterioration of the environment in the fall is inevitable. Living conditions in drying puddles can improve if rain falls and the puddle becomes full again. Then the invertebrates of the embryos preparing for diapause, which have begun preparation for diapause, dissolve. That winter will not be harsh and therefore there is no need to switch to diapause. In response to this second putative signaling factor, the hydrobionts of the embryos preparing for diapause dissolve. In the rain puddles drying up in summer, in which aquatic organisms begin to prepare for diapause, the situation is also different than in water bodies in which the deterioration of the environment in the fall is inevitable. Living conditions in drying puddles can improve if rain falls and the puddle becomes full again. Then the invertebrates of the embryos preparing for diapause, which have begun preparation for diapause, dissolve. The situation is also different than in water bodies in which the deterioration of the environment in the fall is inevitable. Living conditions in drying puddles can improve if rain falls and the puddle becomes full again. Then the invertebrates of the embryos preparing for diapause, which have begun preparation for diapause, dissolve. The situation is also different than in water bodies, in which the deterioration of the environment in the fall is inevitable. Living conditions in drying puddles can improve if rain falls and the puddle becomes full again. Then the invertebrates of the embryos preparing for diapause, which have begun preparation for diapause, dissolve.

Diapause is beneficial only if it occurs before the deterioration of the environment. If it occurs, when, after its onset, the deterioration of the environment does not occur, it brings harm. A population that diapause under favorable conditions is threatened with its displacement from the reservoir by other populations that have not passed into diapause. These other populations use the resources that the diapause population could get if it stopped preparation for diapause in time. Therefore, living in water bodies, in which signaling factors warn of a possible, but not inevitable, deterioration of the environment, requires that aquatic organisms have functional plasticity of the reproductive system, which ensures asexual reproduction. Hydrobionts living in these reservoirs should be capable, having begun to form diapausing somatic embryos, 
interrupt this process and return to the formation of somatic embryos developing without diapause. Resorption of embryos preparing for diapause in modular invertebrates adaptation to the uncertainty of the near future in a reservoir, the living conditions in which can sharply deteriorate, and then, after a short time, quickly become again favorable for life.

\section{About the Mechanism of Interruption of Preparation for Diapause}

A presumptive answer to the question of how the physiological regulatory system works, which controls the resorption of embryos preparing for diapause in sedentary modular invertebrates, can be obtained from plant physiology. In plants, in Unlike sedentary modular animals, the physiological regulatory system governing preparation for seasonal environmental degradation has been studied [10]. Plants, like the invertebrates in question, are modular organisms. Their modules are shoots, leaves, flowers, antennae, with the help of which the climbing species are attached to the support, resting buds. Adaptations to the environment in plants and in modular animals are similar [11]. Resting buds of plants are analogs of diapausing somatic embryos of the named invertebrates. The buds preparing for rest during their growth act on the entire plant, forcing it to give them the nutrients it contains. This ability is called apical dominance. Due to the apical dominance in autumn, the leaves of trees and shrubs are depleted and fall off, in herbaceous plants the aerial part is depleted and dries up in the fall. These disruptions have an adaptive meaning. They provide the replacement of modules that are unstable to seasonal deterioration of the environment with resistant ones - dormant buds. At the same time, resources from dying modules do not disappear, but are used after the reactivation of dormant buds for plant growth in the next favorable season for life. In sedentary modular invertebrates, growing somatic embryos preparing for diapause also draw out nutrients from other parts of the colonial organism. This extension is probably also provided by the apical dominance mechanism. With the resorption of embryos preparing for diapause in the aforementioned invertebrates, the direction of the flow of nutrients is reversed. The embryos preparing for diapause give nutrients, and the tissues of the colony take them.

How is the mechanism of this redirection of the flow of nutrients from one part of the modular organism to others regulated in these invertebrates, and how is the mechanism of apical dominance regulated? Sponges have no nervous and endocrine systems. Therefore, for these mechanisms to work, the participation of the nervous and endocrine systems is not necessary. The growth of embryos preparing for diapause and their resorption, as well as the reversal of the direction of the flow of nutrients in the colony, is controlled by the regulatory physiological system, which is based on intercellular interactions.

\section{Interruption of Preparation for Diapause in Modular Invertebrates and Tumor Regression are Probably the Same Process}

It was said above that resorption of growing embryos preparing for diapause in sessile modular invertebrates and tumor regression are probably the same process. It only manifests itself in different ways in the most low-integrated and in the rest of Metazoa. INIn the course of evolution, Metazoa moved from a modular structure to a unitary one and from a sedentary lifestyle to a freely mobile one. A unitary freely mobile organism is a module of a modular organism that has lost the ability to reproduce asexually. His physiological system, which controlled apical dominance and redirection of the flow of nutrients in distant modular ancestors, was preserved. She began to ensure the course of oncogenesis and tumor regression. With the growth of a tumor (atavistic somatic embryo preparing for diapause), it draws nutrients to itself, and organs and healthy tissues of the body give them away. When the tumor dissolves, it gives up the nutrients it contains. The non-malignant tissues of the convalescent patient pull them towards them. Tumor growth and regression, all of the above is a speculative hypothesis. It is based on the assumption that the first Metazoa on Earth were modular and sedentary. Whether they really were like that paleontologists do not know, since these invertebrates lived long before the Cambrian, when Metazoa did not yet have solid skeletons. Only prints of these invertebrates are known. And fingerprints are not enough to judge the lifestyle of the first Metazoa on Earth. If future studies show that the same mechanism controls the transition to diapause of sessile modular invertebrates and tumorigenesis, it will become clear that the first Metazoa on Earth were indeed modular and sessile. Then everything stated above will become not a hypothesis, but a theory, and the oncologists will have the opportunity to uncover the mechanism of tumor regression by studying the mechanism of resorption in these invertebrates preparing for diapause embryos. And the disclosure of the tumor regression mechanism will make it possible to control it. There will be a possibility of an artificial activation of this mechanism.

\section{References}

1. Ivanova-Kazas OM. Asexual reproduction of animals. L. Ed. Leningrad State University. 1977; 239.

2. Zakhvatkin AA. Comparative embryology of lower invertebrates. 1949; 394.

3. Larson SG. Reflection in the system Deuterostomia. 1963; 2: 5-1228.

4. Beklemishev VN. Fundamentals of comparative anatomy of invertebrates. 1964.

5. Jagersten G. Evolution of the metazoan life cycle. A comprehensive theory. 1972.

6. Marfenin NN. The concept of a modular development organization. Journa of General Biology. 1999.

7. Makrushin AV. Aging and oncogenesis (ontogenetic, evolutionary environmental and social aspects). Peertechz. 2019.

8. Drisch H. Studienüber Regeneration svermōgen der Clavelinalepadiformis. Arch Entw Mech Org. 1902; 14: 227-287.

9. Burighel $P$, Brunetti $R$, Zaniolo $G$. Hibernation of the colonial ascidian, Botrylloidesleachi (Savogni). Boll Zool. 1976; 43: 239-301.

10. Chaikhalyan M. Photoperiodic and hormonal regulation of tuberization in plants. M. Science. 1984; 69.

11. The growth and form of modular organisms. By JL Harper, BR Rosen, $J$ White (editors). Collection of articles by different authors. Phil. Trans Roy Soc London B. 1986; 313: 258. 